\title{
Adubação química, ataque do ácaro Aceria guerreronis e produtividade do coqueiro 'Anão-Verde'
}

\author{
Miguel Michereff Filho ${ }^{(1)}$, Lafayette Franco Sobral(2), Joana Maria Santos Ferreira(2), \\ Agna Rita dos Santos Rodrigues ${ }^{(3)}$ e Mirian Fernandes Furtado Michereff ${ }^{(4)}$
}

\begin{abstract}
(1)Embrapa Recursos Genéticos e Biotecnologia, Caixa Postal 02372, CEP 70770-900 Brasília, DF. E-mail: micher@cenargen.embrapa.br (2)Embrapa Tabuleiros Costeiros, Caixa Postal 44, CEP 49025-040 Aracaju, SE. E-mail: lafayete@cpatc.embrapa.br, joana@cpatc.embrapa.br (3) Universidade Federal Rural de Pernambuco, Departamento de Agronomia, Dom Manoel de Medeiros, s/no, CEP 52171-900 Recife, PE. E-mail: agnarodrigues@yahoo.com.br ${ }^{(4)}$ Universidade de Brasília, Instituto de Ciências Biológicas, Departamento de Zoologia, Campus Darcy Ribeiro, ICC Sul, Asa Norte, CEP 70910-000 Brasília, DF. E-mail: mirianfm@terra.com.br
\end{abstract}

\begin{abstract}
Resumo - O objetivo deste trabalho foi determinar a influência de níveis de nitrogênio e de potássio aplicados em combinação, via fertirrigação, sobre a produtividade do coqueiro 'Anão-Verde', e a intensidade de ataque do ácaro Aceria guerreronis Keifer (Acari: Eriophyidae). O estudo foi conduzido de fevereiro de 2003 a março de 2004, no Município de Neópolis, SE, em um plantio comercial de coqueiro 'Anão-Verde', com oito anos de idade, que desde o ano 2000 vinha recebendo, via microaspersão, os seguintes tratamentos (gramas de $\mathrm{N} \mathrm{e} \mathrm{K}$ por planta por ano): 135 e 135; 1.890 e 1.890; 810 e 135; 135 e 810; 1.890 e 810; e 810 e 1.890. Foram realizadas quatro avaliações trimestrais, entre abril de 2003 e março de 2004, nos cachos de frutos associados às folhas 14 e 18 . A produção de frutos foi influenciada pelos níveis de $\mathrm{N}$ e $\mathrm{K}$ aplicados ao coqueiral, porém a infestação ( 8 a $80 \%$ de frutos atacados) e a severidade dos danos do ácaro-praga (3 a 47\%) não foram afetadas pela adubação química.
\end{abstract}

Termos para indexação: Cocos nucifera, fertirrigação, nitrogênio, nutrição vegetal, potássio.

\section{Chemical fertilization, attack by Aceria guerreronis and productivity of green dwarf coconut}

\begin{abstract}
The objective of this work was to determine the influence of nitrogen and potassium applied through fertigation, on green dwarf coconut productivity, and the intensity of Aceria guerreronis Keifer (Acari: Eriophyidae) attack. The study was carried out in a commercial orchard of eight-year old green dwarf coconut, in the Neópolis County, Sergipe State, Brazil. The orchard received through microsprinkling (fertigation), since 2000, the following treatments (grams per plant per year of $\mathrm{N}$ and $\mathrm{K}$ ): 135 and 135; 1,890 and 1,890; 810 and 135; 135 and 810; 1,890 and 810; and 810 and 1,890. Four evaluations were done every three months between April, 2003 and March, 2004, on fruit bunches associated with the leaves 14 and 18. Fruit production was influenced by $\mathrm{N}$ and $\mathrm{K}$ level applied on the coconut orchard, but mite infestation (from 8 to $80 \%$ of fruits) and the damage severity on fruits (from 3 to $47 \%$ ) were not significantly affected by the chemical fertilization.
\end{abstract}

Index terms: Cocos nucifera, plant nutrition, fertigation, nitrogen, potassium.

\section{Introdução}

O coqueiro Cocos nucifera $\mathrm{L}$. é uma cultura de grande importância socioeconômica para a Região Nordeste do Brasil (Cuenca, 1998). Entre as principais pragas do coqueiro, destaca-se o ácaro-da-necrose-dofruto Aceria guerreronis Keifer (Acari: Eriophyidae). Esse ácaro causa prejuízos significativos à produção do albúmen sólido (copra) na maior parte dos países produtores de coco das Américas do Sul e Central, Antilhas, África e Ásia (Moore \& Howard, 1996). No Brasil, A. guerreronis encontra-se amplamente distribuído nas regiões produtoras de coqueiro, e pode ocasionar elevados níveis de infestação em razão do manejo inadequado do pomar e das condições climáticas (Lawson-Balagbo et al., 2008).

$\mathrm{Na}$ fase adulta, o ácaro mede aproximadamente $255 \mu \mathrm{m}$ de comprimento, possui corpo vermiforme, coloração branco-leitosa ou levemente amarelada e brilhante e apenas dois pares de pernas (Moore \& Howard, 1996). Os frutos atacados por A. guerreronis apresentam clorose triangular a partir das bordas das sépalas, que evolui para necrose marrom-escura com rachaduras longitudinais e aspecto áspero (Moore \& 
Howard, 1996; Ferreira et al., 1998). Além disso, os frutos podem apresentar deformações e cair sem completar o desenvolvimento. Fortes infestações podem reduzir o tamanho e o peso do fruto, o peso do albúmen sólido (copra) e o volume do albúmen líquido (água), além de depreciar o valor comercial do fruto no mercado de coco verde (Ferreira et al., 1998).

A suscetibilidade das culturas aos insetos e ácaros pode ser afetada pelo tipo de adubação utilizada. Tanto o excesso como a carência de nutrientes pode romper o equilíbrio fisiológico da planta e afetar, assim, sua produtividade e resistência/tolerância ao ataque das pragas (Marschner, 1995).

O coqueiro é nutricionalmente exigente, e a quantidade de nutrientes que extrai do solo é alta, uma vez que a planta se desenvolve de forma contínua, com ocorrência simultânea da floração, da frutificação e maturação dos frutos (Ohler, 1999). Conforme Magat (2005), a absorção de $\mathrm{N}$ e de $\mathrm{K}_{2} \mathrm{O}$, pelo coqueiro, chega a 174 e $299 \mathrm{~kg} \mathrm{ha}^{-1}$, respectivamente; destes totais, cerca de $62 \%$ do $\mathrm{Ne}$ e $78 \%$ do K são removidos pelos frutos. Considerando-se que em áreas irrigadas com coqueiro-anão podem ser colhidos 250 frutos por planta por ano, essa remoção de nutrientes pode ser ainda maior, o que torna necessário o manejo adequado das adubações, para não comprometer a produtividade do pomar (Sobral, 1998; Teixeira et al., 2005a). Sobral (2004) observou que a produção de frutos do coqueiro 'Anão-Verde', sob fertirrigação, foi maximizada quando os teores foliares de $\mathrm{N}$ e K corresponderam, respectivamente, a 21,48 e $15,47 \mathrm{~g}^{-1} \mathrm{~kg}^{-1}$.

Atualmente, dispõe-se de informações sobre níveis críticos de nutrientes no solo e na folha de variedades e híbridos do coqueiro, bem como recomendações de adubação para a cultura (Sobral, 1998, 2004; Teixeira et al., 2005a, 2005b); contudo, sabe-se pouco sobre a influência da adubação química na intensidade de ataque deste ácaro aos frutos. Moore et al. (1991), ao comparar o estado nutricional de coqueiro da variedade Gigante e o ataque de A. guerreronis, em Santa Lucia (América Central), verificaram aumento dos danos nos frutos com o incremento no nível de $\mathrm{N}$ nas folhas, enquanto o $\mathrm{K}$ teria efeito negativo. Kannaiyan et al. (2002) relataram redução da infestação do ácaro-da-necrose em coqueiro 'Gigante', adubado com dose elevada de potássio, na Índia. Além disso, nenhum estudo tem abordado esta relação em coqueiros da variedade Anã. Assim, este trabalho teve como objetivo determinar a influência de níveis de $\mathrm{N}$ e de $\mathrm{K}$, aplicados em combinação, via fertirrigação, sobre a produtividade do coqueiro 'Anão-Verde', e a intensidade de ataque do ácaro A. guerreronis.

\section{Material e Métodos}

O estudo foi conduzido no Município de Neópolis, SE, entre março de 2003 e fevereiro de 2004, em pomar de coqueiro 'Anão-Verde', com oito anos de idade e espaçamento de 7,5 m em triângulo eqüilátero, cultivado em Argissolo Amarelo álico (Embrapa, 1999). O clima é do tipo tropical chuvoso, com verão seco e chuvas concentradas nos meses de abril a setembro (Cintra et al., 2004).

O coqueiral vinha recebendo desde 2000 , via fertirrigação, os seguintes níveis de N e K, em gramas por planta por ano: 135:135; 1.890:1.890 [próximo à faixa de adubação recomendada pela pesquisa e assistência técnica (Rosa Júnior et al., 2000; Sobral, 2004; Ferreira Neto, 2005)]; 810:135; 135:810; 1.890:810; e 810:1.890. As fontes utilizadas de $\mathrm{N} \mathrm{e} \mathrm{K}$ foram uréia e $\mathrm{KCl}$, respectivamente. As doses de fertilizantes foram fracionadas em 36 aplicações por ano, uma por semana.

O delineamento experimental foi o de blocos ao acaso, com quatro repetições. Foram utilizadas seis plantas úteis por parcela, localizadas na mesma linha; tendo-se deixado uma fileira sem adubação, como bordadura entre parcelas. O sistema de irrigação consistiu de dois microaspersores autocompensantes por planta (vazão de $40 \mathrm{~L} \mathrm{~h}^{-1}$ por emissor), com aplicação de $150 \mathrm{~L}$ de água por planta por dia. Acaricidas não foram utilizados durante este estudo.

$\mathrm{O}$ estado nutricional das plantas foi determinado mediante análise foliar. Amostras da folha 14 foram coletadas semestralmente nas plantas úteis da cada parcela, tendo-se seguido os procedimentos de Sobral (1998), e os teores foliares de N e K foram determinados de acordo com Silva (1999). Os resultados foram comparados com os níveis críticos de nutrientes para coqueiro 'Anão-Verde', propostos por Sobral (2004).

Foram realizadas quatro avaliações trimestrais do ataque do ácaro, mediante inspeção de frutos nos cachos associados às folhas $14 \mathrm{e} 18$, correspondentes ao quarto e oitavo cacho após a inflorescência mais jovem e plenamente aberta, ao longo da espiral de folhas do coqueiro, conforme proposto por Ferreira et al. (2002). Cronologicamente, em relação à abertura da inflorescência, os cachos amostrados tinham aproximadamente três e seis meses de idade. $\mathrm{O}$ cacho 
da folha 14 foi selecionado por apresentar frutos novos, que poderiam conter elevada população de ácaros, pelo tamanho apropriado do fruto para avaliação visual dos danos na sua superfície (Moore \& Howard, 1996; Fernando et al., 2003), e pela relação desse cacho com a folha utilizada na diagnose do estado nutricional da planta. O cacho da folha 18 , além de conter frutos no ponto de colheita para consumo de água, apresenta frutos com a extensão final dos danos ocasionados pelo ataque do ácaro e seu reflexo na produção.

Para cada cacho inspecionado, determinou-se a percentagem de frutos atacados, a severidade do dano, e a percentagem de frutos comercias, a partir da avaliação do cacho da folha 18. A percentagem de frutos atacados foi obtida pela contagem do número de frutos sadios e de frutos danificados pelo ácaro no cacho. Para avaliação dos danos, utilizou-se escala visual de notas (0 a 4) adaptada de Moore et al. (1989): 0 = fruto sadio; $1=$ clorose e/ou necrose em 1 a $10 \%$ da superfície do fruto; 2 = necrose em 11 a $25 \%$ da superfície do fruto; $3=$ fruto com 26 a $50 \%$ da superfície necrosada, distorção no formato e redução no tamanho; 4 = fruto com 51 a 100\% de superfície necrosada, muito deformado e tamanho muito reduzido. Com os dados obtidos, foi calculado o índice de severidade do dano, pela fórmula de Mckinney (1923):

$$
\mathrm{SD} \%=\frac{\Sigma(\mathrm{n} \times \mathrm{f})}{\mathrm{Z} \times \mathrm{N}} \times 100
$$

em que: SD (\%) é a severidade do dano; $\mathrm{n}$ é a nota da escala conferida ao fruto; f é a freqüência das notas no total das plantas avaliadas; $\mathrm{Z}$ é o valor numérico da nota máxima na escala; e N é o total de observações. Foram considerados como comercializáveis os frutos do cacho da folha 18 que apresentaram danos até a nota 2.

A produção de frutos foi estimada em 12 colheitas, uma por mês, em todas as plantas da parcela, e foi expressa como número de frutos por planta por ano. Os dados foram submetidos à análise de variância, com delineamento em blocos ao acaso e arranjo em parcelas subdivididas, com as épocas de avaliação como subparcela. As médias dos tratamentos foram comparadas pelo teste de Tukey, a 5\% de probabilidade. Dados de produtividade foram correlacionados aos teores foliares de $\mathrm{N}$ e $\mathrm{K}$ obtidos em todos os tratamentos. De forma similar, as características de ataque do ácaro (infestação e severidade de danos), nos cachos de frutos associados à folha 14 , foram correlacionadas aos teores foliares de $\mathrm{N}$ e $\mathrm{K}$, para se determinar a associação entre o estado nutricional da planta e a dinâmica populacional do ácaro.

\section{Resultados e Discussão}

As dosagens crescentes de $\mathrm{N}$ e $\mathrm{K}$ na fertirrigação promoveram aumentos nos teores de $\mathrm{N}$ e $\mathrm{K}$ na folha 14 (Tabela 1). Teores de $\mathrm{N}$ e $\mathrm{K}$ abaixo do nível crítico foram encontrados nos tratamentos com adubação nitrogenada e potássica nas dosagens de $135 \mathrm{~g}$ por planta por ano de $\mathrm{N}$ e de 135 e $810 \mathrm{~g}$ por planta por ano de $\mathrm{K}$, enquanto teores adequados desses nutrientes ocorreram nas parcelas que receberam 810 e $1.890 \mathrm{~g}$ por planta por ano de $\mathrm{N}$ e $1.890 \mathrm{~g}$ por planta por ano de $\mathrm{K}$, respectivamente.

Para uma produção comercial de coco verde, sob condição de irrigação na Região Nordeste, onde se esperam entre 150 a 250 frutos por planta por ano (Rosa Júnior et al., 2000; Ferreira Neto, 2005; Teixeira et al., 2005a), a produtividade média observada, em todos os níveis de adubação testados, foi bastante satisfatória (Tabela 1). A produção de frutos (240-260 frutos por planta por ano), assim como os teores foliares de $\mathrm{N} \mathrm{e} \mathrm{K}$, foram significativamente maiores nas dosagens mais elevadas, correspondentes às proporções de 1.890:1.890 e 1.890:810 g $(\mathrm{N}: \mathrm{K})$ por planta por ano. Isto já era esperado, pois segundo Sobral (2004), a produção de frutos do coqueiro 'Anão-Verde' é maximizada com a aplicação de $1.402 \mathrm{~g}$ de $\mathrm{N}$ por planta por ano e de $1.580 \mathrm{~g}$ de $\mathrm{K}$ por planta por ano. Ferreira Neto (2005) também obteve expressiva produtividade com fertirrigação, nas dosagens de $1.539 \mathrm{~g}$ de $\mathrm{N}$ por planta por ano e de $1.540 \mathrm{~g}$ de K por planta por ano, em coqueiro 'Anão-Verde' com sete anos de idade.

Tabela 1. Teores foliares de nitrogênio e potássio na matéria seca da folha 14 e produção de frutos, em razão da adubação nitrogenada e potássica do coqueiro 'Anão-Verde'(1).

\begin{tabular}{lrrc}
\hline $\begin{array}{l}\text { Nível de N:K } \\
\text { (g por planta por ano) }\end{array}$ & \multicolumn{1}{c}{$\mathrm{N}$} & $\mathrm{K}$ & $\begin{array}{c}\text { Produtividade }^{(2)} \\
\text { (frutos por planta } \\
\text { por ano) }\end{array}$ \\
\hline $135: 135$ & $17,1 \pm 0,4 \mathrm{~b}$ & $9,3 \pm 0,4 \mathrm{c}$ & $203,5 \pm 9,5 \mathrm{~b}$ \\
$1.890: 1.890$ & $21,7 \pm 0,3 \mathrm{a}$ & $15,1 \pm 0,6 \mathrm{a}$ & $260,8 \pm 9,8 \mathrm{a}$ \\
$810: 135$ & $20,6 \pm 0,4 \mathrm{a}$ & $8,5 \pm 0,5 \mathrm{c}$ & $235,5 \pm 8,4 \mathrm{ab}$ \\
$135: 810$ & $17,3 \pm 0,2 \mathrm{~b}$ & $11,3 \pm 0,4 \mathrm{~b}$ & $209,5 \pm 9,0 \mathrm{~b}$ \\
$1.890: 810$ & $21,9 \pm 0,2 \mathrm{a}$ & $11,1 \pm 0,2 \mathrm{~b}$ & $241,8 \pm 9,3 \mathrm{a}$ \\
$810: 1.890$ & $20,7 \pm 0,5 \mathrm{a}$ & $14,9 \pm 0,2 \mathrm{a}$ & $221,8 \pm 9,9 \mathrm{ab}$ \\
\hline
\end{tabular}

(1)Médias ( \pm erro-padrão da média) seguidas por letras iguais, na coluna, não diferem entre si pelo teste de Tukey, a 5\% de probabilidade; $\mathrm{n}=48$ amostras/tratamento; níveis críticos propostos por Sobral (2004): $\mathrm{N}=22 \mathrm{~g} \mathrm{~kg}^{-1}$ e $\mathrm{K}=15 \mathrm{~g} \mathrm{~kg}^{-1}$. ${ }^{(2)}$ Estimativa de produção a partir de 20 plantas amostradas por tratamento. 
Comparativamente, os teores foliares de $\mathrm{N}$ estiveram mais relacionados $(\mathrm{r}=0,72)$ à produtividade do coqueiro do que os teores de $\mathrm{K}(\mathrm{r}=0,41)$, o que indica que, em curto prazo, o crescimento no aporte de $\mathrm{K}$ nas folhas pode não se refletir em aumento na produção de frutos. Isto, provavelmente, ocorreu em razão das grandes quantidades de $\mathrm{K}$ removidas pela colheita dos frutos ainda verdes (Ferreira Neto, 2005).

A percentagem de frutos atacados pelo ácaro e a severidade de dano nos cachos associados às folhas 14 e 18, bem como a percentagem de frutos comerciais, variaram entre as épocas de avaliação (Tabela 2); contudo, a análise de variância não detectou efeito significativo dos níveis de $\mathrm{N}$ e K, utilizados na adubação química do coqueiro, sobre estas variáveis. As perdas na produção em razão do ataque de A. guerreronis variaram de 6 a $20,8 \%$.

Não houve correlação significativa entre as características de ataque do ácaro aos frutos do cacho da folha 14 e os teores foliares de $\mathrm{N}$ e $\mathrm{K}$ obtidos nos diferentes tratamentos. Estes resultados indicam que a população ativa do ácaro sob o perianto dos frutos jovens (cacho da folha 14) e a extensão dos danos no cacho a ser colhido não foram influenciados pela adubação e pelo estado nutricional das plantas.

Resultados obtidos por outros autores, em coqueiro 'Gigante', discordam dos gerados neste trabalho. Este fato pode ser explicado por diferenças intrínsecas da resistência/tolerância ao ácaro e da fisiologia entre as variedades do coqueiro ('Gigante' x 'Anão') avaliadas nos diferentes estudos. Moore et al. (1991) indicam a influência positiva do nitrogênio sobre a infestação de A. guerreronis, porém seu estudo foi essencialmente exploratório e envolveu análise foliar e associação ao ataque do ácaro, em coqueirais da variedade Gigante, sem adoção de delineamento experimental específico para determinar o efeito da adubação sobre a população do ácaro. Muthiah et al. (2001) relataram redução de $52 \%$ nos danos deste ácaro, com o aumento da adubação potássica anual de $1 \mathrm{~kg}$ de $\mathrm{K}_{2} \mathrm{O}$ (830 g de $\left.\mathrm{K}\right)$ para $2 \mathrm{~kg}$ de $\mathrm{K}_{2} \mathrm{O}$ (1.660 g de $\left.\mathrm{K}\right)$ por planta, na variedade Gigante.

$\mathrm{Na}$ Índia, Kannaiyan et al. (2002) recomendaram a aplicação anual de $1,3 \mathrm{~kg}$ de uréia $(585 \mathrm{~g}$ de N) e $3,5 \mathrm{~kg}$ de $\mathrm{KCl}\left(2.096 \mathrm{~g}\right.$ de $\mathrm{K}_{2} \mathrm{O}$ ou $1.740 \mathrm{~g}$ de $\mathrm{K}$ ) por planta, para o manejo do A. guerreronis no coqueiro 'Gigante'. Todavia, verificou-se que a aplicação anual de $1.890 \mathrm{~g}$ de $\mathrm{K}$ por planta não interferiu no ataque do A. guerreronis ao coqueiro 'Anão-verde'. Como a variedade Anã chega a produzir anualmente seis vezes mais frutos (150-300 frutos por planta) que a Gigante (50-80 frutos por planta) (Siqueira et al., 1998; Rosa Júnior et al., 2000), é possível que, para a mesma adubação aplicada, ocorra menor acúmulo de K e de N nos frutos e folhas do coqueiro 'Anão', em comparação ao coqueiro 'Gigante', o que resulta em respostas diferenciadas tanto da planta como da praga.

Tabela 2. Ataque do ácaro Aceria guerreronis, em frutos de cachos das folhas 14 e 18, e percentagem de frutos comerciais, em razão das dosagens de nitrogênio e potássio aplicadas via fertirrigação do coqueiro 'Anão-Verde'.

\begin{tabular}{lccc}
\hline $\begin{array}{l}\text { Dose de N:K } \\
\text { (g por planta por ano) }\end{array}$ & $\begin{array}{c}\text { Frutos atacados } \\
(\%)\end{array}$ & $\begin{array}{c}\text { Severidade de dano } \\
(\%)\end{array}$ & $\begin{array}{c}\text { Frutos comerciais }^{(2)} \\
\text { (frutos por planta) }\end{array}$ \\
\hline & & Cacho da folha 14 & - \\
$135: 135$ & $17,1 \pm 3,6$ & $5,8 \pm 1,4$ & - \\
$1.890: 1.890$ & $15,2 \pm 1,8$ & $6,7 \pm 1,5$ & - \\
$810: 135$ & $15,9 \pm 3,3$ & $5,3 \pm 1,7$ & - \\
$135: 810$ & $19,0 \pm 4,3$ & $7,8 \pm 2,2$ & - \\
$1.890: 810$ & $20,8 \pm 7,1$ & $9,4 \pm 1,9$ & - \\
$810: 1.890$ & $22,5 \pm 5,7$ & $9,2 \pm 1,7$ & $85,5 \pm 4,4$ \\
\hline & & 41,4 & $86,8 \pm 3,7$ \\
$135: 135$ & $73,9 \pm 5,3$ & $40,9 \pm 3,1$ & $84,5 \pm 4,2$ \\
$810: 135$ & $80,0 \pm 4,9$ & $46,9 \pm 2,9$ & $82,7 \pm 5,6$ \\
$135: 810$ & $73,6 \pm 7,2$ & $42,7 \pm 2,3$ & $84,4 \pm 4,0$ \\
$1.890: 810$ & $79,2 \pm 5,9$ & $46,4 \pm 5,6$ & $85,8 \pm 4,9$ \\
$810: 1.890$ & $67,6 \pm 5,8$ & $46,0 \pm 3,3$ & facho da folha 18 (ponto de colheita) \\
\hline
\end{tabular}

${ }^{(1)}$ Médias ( \pm erro-padrão da média) de quatro avaliações trimestrais. (2)Índice de severidade de dano, calculado pela fórmula de Mckinney (1923), a partir de notas de dano de 0 a 4 . ${ }^{(3)}$ Frutos com escala de dano $\leq 2$, ou seja, com 11 a $25 \%$ da superfície do fruto danificada. 
Apesar de a maioria das informações disponíveis demonstrarem que o teor de $\mathrm{N}$ na planta correlaciona-se positivamente com as populações de ácaros-praga e os danos nas plantas (Badegana \& Payne, 2000; Wood \& Relly, 2000), existem outros estudos (Moreira et al., 1999; Sudoi et al., 2001; Leite et al., 2003) que mostram resultados divergentes. A relação entre níveis de $\mathrm{K}$ e ataque das pragas também nem sempre foi tão clara. Segundo Perrenoud (1977), somente grandes suprimentos de K resultaram em decréscimo no ataque das pragas. Isto, provavelmente, pode explicar, pelo menos em parte, os resultados encontrados, uma vez que a nutrição potássica nas dosagens mais elevadas não resultou em teores foliares de $\mathrm{K}$ excedentes, em relação ao nível crítico proposto por Sobral (2004), que corresponde a $15 \mathrm{~g} \mathrm{~kg}^{-1}$ de matéria seca. Além disso, dosagens de K superiores a $1.890 \mathrm{~g}$ de $\mathrm{K}$ por planta por ano, mesmo que fossem eficazes para manejo do ácaro da necrose, poderiam ser tecnicamente inviáveis, porque estariam muito acima do limite de máxima resposta em produção de frutos pelo coqueiro 'Anão-Verde'.

\section{Conclusões}

1. A produção de frutos do coqueiro 'Anão-Verde' é influenciada pelos níveis de $\mathrm{N}$ e $\mathrm{K}$ aplicados via fertirrigação do pomar, e é maior na proporção de 1.890:1.890 g de N:K por planta por ano.

2. A produção de frutos por planta, em coqueiro 'Anão-Verde' com oito anos de idade e irrigado, está positivamente associada aos teores de $\mathrm{N}$ na folha 14 .

3. A percentagem de frutos atacados e a severidade de danos, ocasionadas pelo ácaro Aceria guerreronis, bem como a percentagem de frutos comerciais, não são afetadas pelas adubações nitrogenada e potássica no coqueiro 'Anão-Verde'.

\section{Referências}

BADEGANA, A.M.; PAYNE, V.K. The effect of leaf contents of N, $\mathrm{P}, \mathrm{K}, \mathrm{Ca}$ and $\mathrm{Mg}$ nutrients on the population of the two-spotted spider mite Tetranychus urticae Koch (Acari: Tetranychidae). Mededelingen Faculteit Landbouwkundige en Toegepaste Biologische Wetenschappen Universiteit Gent, v.65, p.221-226, 2000.

CINTRA, F.L.D.; PORTELA, J.C.; NOGUEIRA, L.C. Caracterização física e hídrica em solos dos Tabuleiros Costeiros no distrito de irrigação Platô de Neópolis. Revista Brasileira de Engenharia Agrícola e Ambiental, v.8, p.45-50, 2004.
CUENCA, M.A.G. Importância econômica do coqueiro. In: FERREIRA, J.M.S.; WARWICK, D.R.N.; SIQUEIRA, L.A. (Ed.). A cultura do coqueiro no Brasil. 2.ed. Brasília: Embrapa-SPI; Aracaju: Embrapa-CPATC, 1998. p.17-56.

EMBRAPA. Centro Nacional de Pesquisa de Solos. Sistema de classificação de solos. Brasília: Embrapa-SPI; Rio de Janeiro: Embrapa-CNPS, 1999. 412p.

FERNANDO, L.C.P.; ARATCHIGE, N.S.; PEIRIS, T.S.G. Distribution patterns of coconut mite, Aceria guerreronis, and its predator Neoseiulus aff. paspalivorus in coconut palms. Experimental and Applied Acarology, v.31, p.71-78, 2003.

FERREIRA, J.M.S.; LIMA, M.F.; SANTANA, D.L.; MOURA, J.I.L.; SIQUEIRA, L.A. de. Pragas do coqueiro. In: FERREIRA, J.M.S.; WARWICK, D.R.N.; SIQUEIRA, L.A. (Ed.). A cultura do coqueiro no Brasil. 2.ed. Brasília: Embrapa-SPI; Aracaju: EmbrapaCPATC, 1998. p.189-267.

FERREIRA, J.M.S.; MICHEREFF FILHO, M.; LINS, P.M.P. Monitoramento fitossanitário da plantação do coqueiro. In: FERREIRA, J.M.S.; MICHEREFF FILHO, M. (Org.). Produção Integrada de Coco: práticas fitossanitárias. Aracaju: Embrapa Tabuleiros Costeiros, 2002. p.11-35.

FERREIRA NETO, M. Doses de N e K aplicadas via fertirrigação na cultura do coqueiro (Cocos nucifera L.) 'Anão'. 2005. 105p. Tese (Doutorado) - Escola Superior de Agricultura Luiz de Queiroz, Piracicaba.

KANNAIYAN, S.; RABINDRA, R.J.; RAMARAJU, K.; DORAISWAMY, S. Integrated management of eriophyid mite on coconut. Coimbatore: Tamil Nadu Agricultural University, 2002. $82 \mathrm{p}$.

LAWSON-BALAGBO, L.M.; GONDIM JÚNIOR, M.G.C.; MORAES, G.J.; HANNA, R.; SCHAUSBERGER, P. Exploration of the acarine fauna on coconut palm in Brazil with emphasis on Aceria guerreronis (Acari: Eriophyidae) and its natural enemies. Bulletin of Entomological Research, v.98, p.83-96, 2008.

LEITE, G.L.D.; PICANÇO, M.; ZANUNCIO, J.C.; MARQUINI, F. Factors affecting mite herbivory on eggplants in Brazil. Experimental and Applied Acarology, v.31, p.243-252, 2003.

MAGAT, S.S. World fertilizer use manual: coconut. Disponível em: http://www.fertilizer.org/ifa/publicat/html/pubman/coconut.htm. Acesso em: 11 abr. 2005.

MARSCHNER, H. Mineral nutrition of higher plants. 2.ed. New York: Academic Press, 1995. 889p.

MCKINNEY, H.H. Influence of soil temperature and moisture on infection of wheat seedlings by Helminthosporium sativum. Journal of Agricultural Research, v.26, p.195-218, 1923.

MOORE, D.; ALEXANDER, L.; HALL, L.A. The coconut mite, Eriophyes guerreronis Keifer in St. Lucia: yield losses and attempts to control it with acaricide, polybutene and Hirsutella thompsonii. Tropical Pest Management, v.35, p.83-89, 1989.

MOORE, D.; HOWARD, F.W. Coconuts. In: LINDQUIST, E.E.; SABELIS, M.W.; BRUIN, J. (Ed.). Eriophyoid mites: their biology, natural enemies and control. Amsterdam: Elsevier Science, 1996. p.561-570. 
MOORE, D.; RIDOUT, M.S.; ALEXANDER, L. Nutrition of coconuts in St. Lucia and relationship with attack by coconut mite Aceria guerreronis Keifer. Tropical Agriculture, v.68, p.41-44, 1991.

MOREIRA, A.N.; OLIVEIRA, J.V.; HAJI, F.N.P.; PEREIRA, J.R. Efeito de diferentes níveis de NPK na infestação de Aculops lycopersici (Massee) (Acari: Eriophyidae), em tomateiro no Submédio do Vale do São Francisco. Anais da Sociedade Entomológica do Brasil, v.28, p.275-284, 1999.

MUTHIAH, C.; BHASKARAN, R.; KANNAIYAN, S. Bio-ecology and control of eriophyid mite of coconut: an Indian experience. Planter, v.77, p.255-263, 2001.

OHLER, J.G. Modern coconut management: palm cultivation and products. London: Intermediate Technology Publications, 1999. 458p.

PERRENOUD, S. Potassium and plant health. Bern: International Potash Institute, 1977. 201p.

ROSA JÚNIOR, C.D.R.M.; VIEIRA, V.J.S.; MELO, J.J.S.; SILVA FILHO, A.V. Coqueiro (Cocos nucifera): cultivo sob condição irrigada. 2.ed. Recife: Sebrae/PE, 2000. 50p.

SILVA, F.C. da. Manual de análises químicas de solos, plantas e fertilizantes. Brasília: Embrapa Comunicação para Transferência de Tecnologia; Rio de Janeiro: Embrapa Solos; Campinas: Embrapa Informática Agropecuária, 1999. 370p.

SIQUEIRA, E.R. de; RIBEIRO, F.E.; ARAGÃO, W.M.; TUPINAMBÁ, E.A. Melhoramento genético do coqueiro. In:
FERREIRA, J.M.S.; WARWICK, D.R.N.; SIQUEIRA, L.A. (Ed.). A cultura do coqueiro no Brasil. 2.ed. Brasília: Embrapa-SPI; Aracaju: Embrapa-CPATC, 1998. p.73-98.

SOBRAL, L.F. Fertirrigação do coqueiro 'Anão-Verde' com N e K no Platô de Neópolis. In: FERTIBIO, 26., 2004, Lages. Anais. Lages: Udesc, 2004. CD-ROM.

SOBRAL, L.F. Nutrição e adubação do coqueiro. In: FERREIRA, J.M.S.; WARWICK, D.R.N.; SIQUEIRA, L.A. (Ed.). A cultura do coqueiro no Brasil. 2.ed. Brasília: Embrapa-SPI; Aracaju: Embrapa-CPATC, 1998. p.129-157.

SUDOI, V.; KHAEMBA, B.M.; WANJALA, F.M.E. Nitrogen fertilization and yield losses of tea to red crevice mite (Brevipalpus phoenicis Geijskes) in the Eastern Highlands of Kenya. International Journal of Pest Management, v.47, p.207-210, 2001.

TEIXEIRA, L.A.J.; BATAGLIA, O.C.; BUZETTI, S.; FURLANI JÚNIOR, E. Adubação com NPK do coqueiro 'AnãoVerde' (Cocos nucifera L.) - atributos químicos do solo e nutrição da planta. Revista Brasileira de Fruticultura, v.27, p.115119,2005 a.

TEIXEIRA, L.A.J.; BATAGLiA, O.C.; BUZETTI, S.; FURLANI JÚNIOR, E. Recomendação de adubação e calagem para coqueiro (Cocos nucifera L.) no Estado de São Paulo $1^{\text {a }}$ aproximação. Revista Brasileira de Fruticultura, v.27, p.519-520, 2005b.

WOOD, B.W.; REILLY, C.C. Pest damage to pecan is affected by irrigation, nitrogen application, and fruit load. HortScience, v.35, p.669-672, 2000.

$\overline{\text { Recebido em } 18 \text { de setembro de } 2007 \text { e aprovado em } 3 \text { de março de } 2008}$ 